\title{
Regret in the On-Line Decision Problem
}

\author{
Dean P. Foster \\ Department of Statistics, The Wharton School, University of Pennsylvania, \\ Philadelphia, Pennsylvania 19104
}

and

Rakesh Vohra

Department of Managerial Economics and Decision Sciences, Kellogg School of Management, Northwestern University,

Evanston, Illinois 60208

Received A pril 12, 1999

\begin{abstract}
At each point in time a decision maker must make a decision. The payoff in a period from the decision made depends on the decision as well as on the state of the world that obtains at that time. The difficulty is that the decision must be made in advance of any knowledge, even probabilistic, about which state of the world will obtain. A range of problems from a variety of disciplines can be framed in this way. In this paper we survey the main results obtained, as well as some of their applications. Journal of Economic Literature Classification N umbers: C 70, C 73.

(c) 1999 A cademic Press
\end{abstract}

\section{INTRODUCTION}

At each (discrete) point in time a decision maker must make a decision. The loss (or reward) from the decision made depends on the decision and the state of the world that obtains at that time. If $d_{t}$ is the decision made at time $t$ and $X_{t}$ is the state of the world at time $t$, the loss incurred is $L\left(d_{t}, X_{t}\right)$ and is nonnegative and bounded. The catch is that the decision must be made prior to knowing anything about which state of the world will obtain. The decision maker's goal is to select a sequence of decisions $\left\{d_{t}\right\}_{t \geq 0}$ such that his or her total loss,

$$
\sum_{t=0}^{T} L\left(d_{t}, X_{t}\right)
$$


is small. We call this the on-line decision problem (ODP). The decision maker's goal as we have described it is not well defined. We return to this issue later in this section. ODPs are different from many of the online problems considered in computer science in that the loss incurred in each period does not depend on decisions taken in earlier periods. The interested reader should consult I rani and Karlin (1996) for a brief survey of work on on-line problems in computer science.

A range of problems from a variety of disciplines can be framed as ODPS. O ne example of an ODP that has received much attention is the problem of predicting a sequence of 0 's and 1 's in such a way as to minimize the number of incorrect predictions (see, for example, Cesa-Bianchi et al., 1993 or Vovk, 1990). In this case there are two possible decisions in each time period, predict a 1 or predict a 0 ; i.e., $d_{t}=0,1$. In each time period there are just two possible states of the world, 0 or 1 ; i.e., $X_{t}=0,1$. The loss function will be $L\left(d_{t}, X_{t}\right)=\left|d_{t}-X_{t}\right|$. Other examples will be mentioned in the body of the paper when appropriate. ODPs have been the subject of study for over 40 years now in statistics, computer science, game theory, information theory, and finance. Furthermore, investigations in these different disciplines have been pursued quite independently. O ne measure of this is that one particular result (which we will describe) has been proved independently on at least four different occasions within this 40-year span!

We turn now to the important issue of what the decision maker's goal is. E arlier we said it was to minimize the total loss. The problem is that the loss will depend on the particular sequence of states of the world that transpire. For example, consider the $0-1$ prediction problem mentioned earlier. Here is a naive prediction scheme: predict 1 every time. If the sequence that obtained were all 1, then we would be in the pleasant position of having the smallest possible loss, 0 . D oes this mean that this prediction scheme is a good one? Clearly not. If the sequence being predicted had been all 0 's, the scheme would definitely be useless. What is needed is a scheme that generates low average losses against a variety of sequences of states of the world. O ne natural way of operationalizing the robustness requirement is to focus on

$$
\max \sum_{t=0}^{T} L\left(d_{t}, X_{t}\right) .
$$

Here the maximum is over all possible sequences of states of the world. The goal is to find a scheme for selecting decisions that minimizes this last quantity. This goal, while well defined, is not useful. Consider the 0-1 prediction problem again. For every deterministic prediction scheme there is a sequence of 0 's and 1's for which the scheme never makes a correct prediction. So, the maximum over all sequences of the time-averaged loss for every deterministic prediction scheme is 1 . 
If one is not wedded to deterministic prediction schemes, there is an obvious way out, and that is to randomize. Thus, $\sum_{t=0}^{T} L\left(d_{t}, X_{t}\right)$ becomes a random variable. In this case, one natural definition of robustness is

$$
\max E\left[\sum_{t=0}^{T} L\left(d_{t}, X_{t}\right)\right],
$$

where the expectation is with respect to the probabilities induced by the randomized scheme. In this paper we restrict our attention to randomized schemes only.

U nfortunately, the best that can be achieved by a randomized scheme is an average loss of $1 / 2$ per round. This is obtained when the decision maker randomizes $50 / 50$ on each round. Since finding a scheme that has $\max E\left[\sum_{t=0}^{T} L\left(d_{t}, X_{t}\right)\right]$ less than $T / 2$ is impossible, an alternative has been (see, for example, Cesa-Bianchi et al., 1993; Cover, 1991; Foster and Vohra, 1993) to measure the success of a decision scheme by comparison with other schemes. Imagine that we have a family $\mathscr{F}$ of decision schemes already available. Let $S$ be a new scheme. $O$ ne would view $S$ as being attractive if its total loss were "comparable" to the total loss of the best scheme in $\mathscr{F}$, no matter what sequence of states of the world obtains.

The comparability idea judges a scheme on the basis of a notion of external validity; i.e., is it as good as some other scheme? In this paper we introduce an alternative to this, which judges a scheme on the basis of its internal coherence. We also establish a close connection between this notion of internal coherence and one version of comparability, allowing us to derive several known results in a unified way.

\section{REGRET}

R egret is what we feel when we realize that we would have been better off had we done something else. A basic requirement of any scheme is that it should avoid or at least reduce the regret that will be felt. B efore we give an explicit definition, we introduce some notation. ${ }^{1}$ Let $D=\left\{d_{1}, d_{2}, \ldots, d_{n}\right\}$ be the set of possible decisions in each time period. ${ }^{2}$ D enote the loss incurred at time $t$ from taking decision $d_{j}$ by $L_{t}^{j}$. We assume throughout that losses are bounded; in fact, to save on notation, assume that $L_{t}^{j} \leq 1$ for all $d_{j} \in D$ and $t \geq 0$. Notice that we suppress the dependence on the state of the world that obtains at time $t$.

${ }^{1}$ There can be many ways to operationalize the notion of regret; we offer only one.

${ }^{2}$ The analysis can easily be extended to the case of different sets of decisions at each time period at the cost of increased notation. 
A ny scheme (deterministic or randomized) for selecting decisions can be described in terms of the probability, $w_{t}^{j}$, of choosing decision $j$ at time $t$. Let $w_{t}$ denote the $n$-tuple of probabilities at time $t$. Remember, $w_{t}$ must be derived using only data obtained up to time $t-1$.

Consider now a scheme $S$ for selecting decisions. Let $\left\{w_{t}\right\}_{t \geq 0}$ be the probability weights implied by the scheme. Then, the expected loss from using $S, L(S)$, over $T$ periods will be

$$
\sum_{t=1}^{T} \sum_{d_{j} \in D} w_{t}^{j} L_{t}^{j} .
$$

I magine we have applied the scheme $S$ for $T$ periods. Now, we look back and review our performance. $\mathrm{H}$ ad we done things differently, could we have wound up with a smaller loss? Specifically, at every time $t$ that the scheme $S$ said we should pick decision $d_{j}$ with probability $w_{t}^{j}$, had we picked decision $d_{i}$ would we have done better? $\mathrm{H}$ ad we done so, our expected loss would be

$$
L(S)-\left(\sum_{t=1}^{T} w_{t}^{j} L_{t}^{j}-\sum_{t=1}^{T} w_{t}^{j} L_{t}^{i}\right) .
$$

If the quantity

$$
\sum_{t=1}^{T} w_{t}^{j} L_{t}^{j}-\sum_{t=1}^{T} w_{t}^{j} L_{t}^{i}
$$

were positive, then clearly we would have been better off. So we feel regret at having made decision $d_{j}$ instead of decision $d_{i}$. For this reason we define the regret incurred by $S$ from using decision $d_{j}$ to be

$$
R_{T}^{j}(S)=\sum_{i \in D} \max \left\{0,\left(\sum_{t=1}^{T} w_{t}^{j}\left(L_{t}^{j}-L_{t}^{i}\right)\right)\right\} .
$$

The regret from using $S$ will be

$$
R_{T}(S)=\sum_{j \in D} R_{T}^{j}(S) .
$$

The scheme $S$ will have the no internal regret property if its expected regret is small, i.e.,

$$
R_{T}(S)=o(T) .
$$

Notice that a no internal regret scheme has a (time) averaged regret that goes to zero as $T \rightarrow \infty$, i.e., $R_{T}(S) / T \rightarrow 0$. The existence of a no internal regret scheme was first established by Foster and Vohra (1998). The proof we describe here is due to $\mathrm{H}$ art and $\mathrm{M}$ as-Collel (1996) and makes use of 
D avid Blackwell's approachability theorem. For completeness we include a statement and proof of the approachability theorem in an appendix to this paper.

A ctually we will prove something stronger. We show that the time average of the realized regret will go to zero almost surely as $T \rightarrow \infty$. To motivate the proof we consider the case $|D|=2$ first.

\subsection{The Case $|D|=2$}

Let $I(j, t)$ be an indicator variable that is equal to 1 if decision $d_{j}$ was chosen at time $t$. Given any two decisions $d_{i}$ and $d_{j}$, define the realized pairwise regret of switching from $d_{j}$ to $d_{i}$ to be

$$
R_{T}^{j \rightarrow i}(S)=\sum_{t=1}^{T} I(j, t) L_{t}^{j}-\sum_{t=1}^{T} I(j, t) L_{t}^{i} .
$$

Our goal is to show that there is a randomized scheme $S$ such that $R_{T}^{j \rightarrow i}(S) / T \rightarrow 0$ almost surely as $T \rightarrow \infty$. Notice that the expected value of $R_{T}^{j \rightarrow i}(S)$ is $\sum_{t=1}^{T} w_{t}^{j}\left[L_{t}^{j}-L_{t}^{i}\right]$, so it clearly follows that there is a randomized scheme $S$ such that $\max _{j} R_{T}^{j}(S)=o(T)$. If this last statement is true, it will follow that $R_{T}(S)=o(T)$.

Since $R_{T}^{i \rightarrow i}(S)$ is zero, if $|D|=2$ we only have two nontrivial component regrets, $R_{T}^{1 \rightarrow 0}(S)$ and $R_{T}^{0 \rightarrow 1}(S)$. If we can choose the decisions in each round in such a way as to force the time average of $R_{T}^{1 \rightarrow 0}(S)$ and $R_{T}^{0 \rightarrow 1}(S)$ to go to zero, we are done.

To use the approachability theorem we need to define both a game and a target set. In the game the decision maker has one strategy for each decision. The payoff from using strategy " 0 " at time $t$ is the vector $\left(L_{t}^{0}-L_{t}^{1}, 0\right)$, while the vector payoff from using strategy " 1 " at time $t$ is $\left(0, L_{t}^{1}-L_{t}^{0}\right)$. Suppose the decision maker uses a scheme $S$ that selects strategy " 0 " with probability $w_{t}$ in round $t$. Then his or her realized time-averaged (vector) payoff after $T$ rounds will be

$$
\left(\frac{\sum_{t=1}^{T} w_{t}\left[L_{t}^{0}-L_{t}^{1}\right]}{T}, \frac{\sum_{t=1}^{T}\left(1-w_{t}\right)\left[L_{t}^{1}-L_{t}^{0}\right]}{T}\right),
$$

which is just $\left(R_{T}^{0 \rightarrow 1}(S) / T, R_{T}^{1 \rightarrow 0}(S) / T\right)$. Given what we wish to prove, the target set is simply the nonpositive orthant. Figure 1 shows a picture of this situation. The line $l$ is the line through the origin whose normal is $\left(\left[R_{T}^{0 \rightarrow 1}(S) / T\right]^{+},\left[R_{T}^{1 \rightarrow 0}(S) / T\right]^{+}\right)$.

Blackwell's approachability theorem tells us that if the decision maker can find a strategy which forces the vector payoff (in expectation) to be on the same side of the line $l$ as the target set, she can force the long-term 


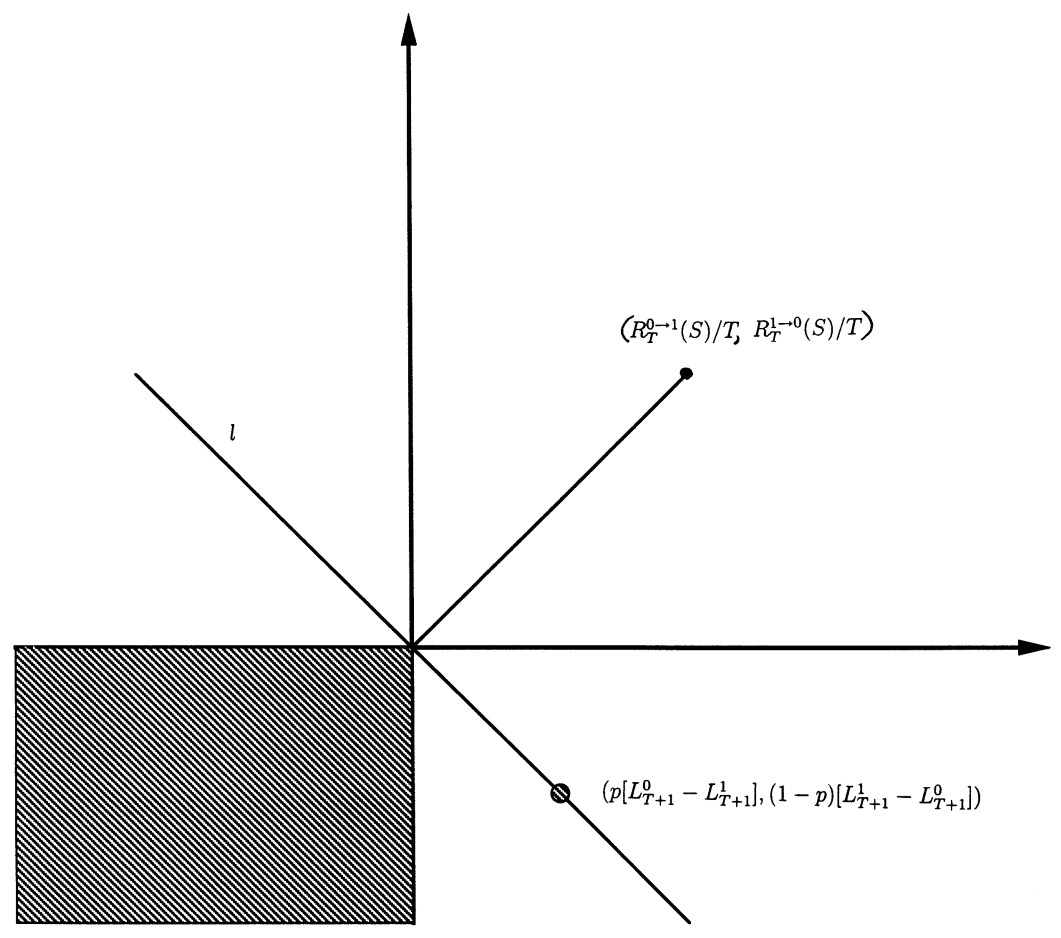

G

FIGURE 1

average of the payoffs to be arbitrarily close to the target set. If the average of the payoffs is already in the target set, we are done.

We now show that it is possible to force the next vector payoff to lie on the same side of line $l$ as the target set. A fter $T$ rounds the time-averaged payoff is the vector $\left(R_{T}^{0 \rightarrow 1}(S) / T, R^{1 \rightarrow 0}(S) / T\right)$. Thus the equation of the line $l$ will be $\left[R_{T}^{0 \rightarrow 1}(S) / T\right]^{+} x+\left[R_{T}^{1 \rightarrow 0}(S) / T\right]^{+} y=0$.

If the decision maker chooses strategy 0 with probability $p$ and strategy 1 with probability $1-p$ in round $T+1$, the payoff (in expectation) will be the vector

$$
\left(p\left[L_{T+1}^{0}-L_{T+1}^{1}\right],(1-p)\left[L_{T+1}^{1}-L_{T+1}^{0}\right]\right) .
$$

It suffices to choose $p$ so that this point lies on the line $l$, i.e.,

$\left[R_{T}^{0 \rightarrow 1}(S) / T\right]^{+} p\left[L_{T+1}^{0}-L_{T+1}^{1}\right]+\left[R_{T}^{1 \rightarrow 0}(S) / T\right]^{+}(1-p)\left[L_{T+1}^{1}-L_{T+1}^{0}\right]=0$.

Notice-and this is the important point-that the terms involving $T+1$ cancel, yielding

$$
p\left(R_{T}^{1 \rightarrow 0}(S)\right)^{+}=(1-p)\left(R_{T}^{0 \rightarrow 1}(S)\right)^{+} .
$$


To verify that the value of $p$ that solves this equation is between 0 and 1 , we solve the equation

$$
p=\frac{\left(R_{T}^{0 \rightarrow 1}(S)\right)^{+}}{\left(R_{T}^{1 \rightarrow 0}(S)\right)^{+}+\left(R_{T}^{0 \rightarrow 1}(S)\right)^{+}} .
$$

Notice that the required $p$ depends only on the regrets up to time $T$ and no farther.

\subsection{General Case}

In the general case, where $|D|=n$, there are a total of $n(n-1)$ nontrivial pairwise regret terms. As before, we will identify a scheme $S$ such that $R_{T}^{i \rightarrow j}(S)=o(T)$ for all $i$ and all $j$. Such a scheme will obviously have the no internal regret property.

The proof mimics the $|D|=2$ case. The decision maker has one strategy for every decision. The payoff from playing strategy $j$ in round $T$ is an $n^{2}$ dimensional vector. The first $n(j-1)$ components are 0 , components $(j-$ 1) $n+r$ for $1 \leq r \leq n$ are equal to $L_{T}^{j}-L_{T}^{r}$, and all remaining components are 0 . The target set is $G=\left\{x \in \Re^{n^{2}} \mid(\forall i) x_{i} \leq 0\right\}$.

Call the time average of the vector payoffs obtained so far $a$. N otice that its components will be of the form $\left(R_{T}^{j \rightarrow i}(S) / T\right)_{i, j}$. Let $c$ be the point in $G$ closest to $a$. Clearly, $c_{i}=a_{i}^{-}$. Thus the vector $a-c$ is just $a_{i}^{+}$.

In the next round we want to choose a probability vector $w_{T+1}$, so that the expected vector payoff will lie on the plane $l$, which is perpendicular to $a-c$. Thus, $w_{T+1}$ must satisfy

$$
\sum_{i, j} w_{T+1}^{i}\left(L_{T+1}^{i}-L_{T+1}^{j}\right)\left(R_{T}^{i \rightarrow j}(S)\right)^{+}=0 .
$$

Splitting it into two sums,

$$
\sum_{i, j} w_{T+1}^{i} L_{T+1}^{i}\left(R_{T}^{i \rightarrow j}(S)\right)^{+}-\sum_{i, j} w_{T+1}^{i} L_{T+1}^{j}\left(R_{T}^{i \rightarrow j}(S)\right)^{+}=0 .
$$

Changing the indices of the second sum,

$$
\sum_{i, j} w_{T+1}^{i} L_{T+1}^{i}\left(R_{T}^{i \rightarrow j}(S)\right)^{+}-\sum_{j, i} w_{T+1}^{j} L_{T+1}^{i}\left(R_{T}^{j \rightarrow i}(S)\right)^{+}=0,
$$

we get

$$
\sum_{i, j} L_{T+1}^{i}\left(w_{T+1}^{i}\left(R_{T}^{i \rightarrow j}(S)\right)^{+}-w_{T+1}^{j}\left(R_{T}^{j \rightarrow i}(S)\right)^{+}\right)=0 .
$$

Since the $L_{T+1}^{i}$ 's are arbitrary, we must have for each $i$ that

$$
\sum_{j} w_{T+1}^{i}\left(R_{T}^{i \rightarrow j}(S)\right)^{+}-w_{T+1}^{j}\left(R_{T}^{j \rightarrow i}(S)\right)^{+}=0 .
$$


To complete the argument it suffices to show that this system of equations admits a nonnegative solution.

Let $A$ be a matrix defined as

$$
a_{i j}=R_{T}^{j \rightarrow i}(S)
$$

for all $i \neq j$, and

$$
a_{i i}=-\sum_{j \neq i} R_{T}^{i \rightarrow j}(S) .
$$

Notice that the row sums of $A$ are all zero. Equation (7) is equivalent to $A x=0$. We need to show that the system $A x=0$ admits a nontrivial nonnegative solution. ${ }^{3}$

Let $A^{\prime}$ be the matrix obtained from $A$ as

$$
a_{i j}^{\prime}=a_{i j} / B \text {, }
$$

where $B=\max _{i, j}\left|a_{i j}\right|$. N otice that $\left|a_{i j}^{\prime}\right| \leq 1$ and $\sum_{i} a_{i j}^{\prime}=0$. Let $P=A^{\prime}+$ $I$. Then $P$ will be a nonnegative row stochastic matrix. Hence there is a nonnegative probability vector $x$ such that $P x=x$ (since we do not require that $x$ be unique, we do not need any restrictions on the matrix $P$ ). Since $P=A^{\prime}+I$ we deduce that

$$
\begin{aligned}
& A^{\prime} x+I x=x \\
& \Rightarrow A^{\prime} x=0 \\
& \Rightarrow A x=0
\end{aligned}
$$

The vector $x$ is the required distribution. Further, it can easily be found by Gaussian elimination.

With some additional effort one can extract the rate of convergence of $R_{T}(S)$. It is $O(\sqrt{T})$, and this is the best possible. H owever, for special cases it can be improved.

\subsection{Calibrated Forecasts}

Probability forecasting is the act of assigning probabilities to an uncertain event. There are many criteria for judging the effectiveness of a probability forecast. The one that we consider is called calibration. In this section we will show how the existence of a no internal regret decision scheme implies the existence of a close to calibrated probability forecast. This was first established by Foster and Vohra (1998).

For simplicity, assume that we are forecasting a sequence of $0-1$ 's; i.e., there are just two states of the world. Let $X$ be a sequence of $0-1$ 's whose

${ }^{3}$ The solution can be normalized to turn it into a probability vector. 
$i$ th element is $X_{i}$. Fix a forecasting scheme $F$, and let $f_{i}$ be the probability forecast of a 1 in period $i$ generated by this scheme. Note that $f_{i}$ can be any number between 0 and 1 . Let $n_{t}(p, X, F)$ be the number of times up to time $t$ that the scheme forecasted a probability $p$ of a 1 . L et $\rho_{t}(p, X, F)$ be the fraction of those times that it actually rained. In other words,

$$
\begin{aligned}
& n_{t}(p, X, F) \equiv \sum_{i=1}^{t} I_{f_{i}=p} \\
& \rho_{t}(p, X, F) \equiv \sum_{i=1}^{t} \frac{I_{f_{i}=p} X_{i}}{n_{t}(p, X, F)},
\end{aligned}
$$

where $I$ is the indicator function. The calibration score of $F$ with respect to the sequence $X$ of 0 -1's after $t$ periods is

$$
C_{t}(F, X)=\sum_{p}\left(\rho_{t}(p, X, F)-p\right)^{2} \frac{n_{t}(p, X, F)}{t} .
$$

I deally, one would like an $F$ such that $C_{t}(F, X)=0$ for all $t$ and $X$; i.e., $F$ is calibrated with respect to all sequences $X$. This is impossible, ${ }^{4}$ so, we settle for something less: find a randomized $\mathrm{F}$ such that for any $\epsilon>0$ and all $X$ there is a $t$ sufficiently large so that $C_{t}(F, X)<\epsilon$ almost surely (where the "almost surely" is with respect to the probabilities induced by $F$ ). Here we will establish something weaker.

We restrict $F$ to choosing a forecast from the set $\{0,1 / k, 2 / k, \ldots,(k-$ $1) / k, 1\}$. Let $w_{t}^{j}$ be the probability that $F$ selects the forecast $j / k$ in period $t$. Hence the expected number of times that $F$ chooses $j / k$ as a forecast up to time $t$ is $\sum_{s=1}^{t} w_{s}^{j}$. Let

- $\tilde{n}_{t}(i / k) \equiv \sum_{s=1}^{t} w_{s}^{i}$ and

- $\tilde{\rho}_{t}(i / k) \equiv \sum_{s=1}^{t} w_{s}^{i} I_{X_{s}} / \tilde{n}_{t}(i / k)$.

Let

$$
\tilde{C}_{t} \equiv \sum_{j=0}^{k} \frac{\tilde{n}_{t}(j / k)}{t}\left(\tilde{\rho}_{t}\left(\frac{j}{k}\right)-\frac{j}{k}\right)^{2} .
$$

O ne can think of $\tilde{C}_{t}$ as something akin to an expected calibration score. ${ }^{5}$ We establish the existence of a forecasting scheme with the property that $\tilde{C}_{t} \rightarrow 0$ as $t \rightarrow \infty$.

Consider the following loss function: $L_{t}^{j}=\left(X_{t}-\frac{j}{k}\right)^{2}$. We claim that if $F$ is chosen to be a no internal regret decision scheme with respect to the

${ }^{5}$ In Foster and Vohra (1998) it is shown that $C_{t}(F, X)-\tilde{C}_{t} \rightarrow 0$ almost surely as $t \rightarrow \infty$. 
loss function just defined, then $\tilde{C}_{t} \rightarrow 0$ as $t \rightarrow \infty$. The idea is to show that $\tilde{C}_{t}=O\left(R_{t}(F) / t\right)$.

Let

$$
a_{t}(i, j)=\sum_{s} w_{s}^{i}\left(X_{s}-\tilde{\rho}_{t}\left(\frac{j}{k}\right)\right)^{2}
$$

A straightforward but tedious calculation establishes

$$
\begin{aligned}
a_{t}(i, i)-a_{t}(i, j)= & \tilde{n}_{t}\left(\frac{i}{k}\right)\left(\tilde{\rho}_{t}\left(\frac{i}{k}\right)-\frac{i}{k}\right)^{2} \\
& -\tilde{n}_{t}\left(\frac{i}{k}\right)\left(\tilde{\rho}_{t}\left(\frac{i}{k}\right)-\frac{j}{k}\right)^{2} .
\end{aligned}
$$

Rewriting this, we obtain

$$
\tilde{n}_{t}\left(\frac{i}{k}\right)\left(\tilde{\rho}_{t}\left(\frac{i}{k}\right)-\frac{i}{k}\right)^{2}=a_{t}(i, i)-a_{t}(i, j)+\tilde{n}_{t}\left(\frac{i}{k}\right)\left(\tilde{\rho}_{t}\left(\frac{i}{k}\right)-\frac{j}{k}\right)^{2} .
$$

Since this must be true for all $j$, it must be true for the $j^{*}$ that minimizes $\tilde{n}_{t}(i / k)\left(\tilde{\rho}_{t}(i / k)-j / k\right)^{2}$. Notice that $j^{*}$ can be chosen so that $j^{*} / k$ is within $1 /(2 k)$ of $\tilde{\rho}_{t}(j)$. Hence

$$
\begin{aligned}
\tilde{n}_{t}\left(\frac{i}{k}\right)\left(\tilde{\rho}_{t}\left(\frac{i}{k}\right)-\frac{i}{k}\right)^{2} & \leq a_{t}(i, i)-a_{t}\left(i, j^{*}\right)+\frac{\tilde{n}_{t}(i / k)}{4 k^{2}} \\
& \leq a_{t}(i, i)-\min _{j} a_{t}(i, j)+\frac{\tilde{n}_{t}(i / k)}{4 k^{2}} .
\end{aligned}
$$

Thus

$$
\tilde{n}_{t}\left(\frac{i}{k}\right)\left(\tilde{\rho}_{t}\left(\frac{i}{k}\right)-\frac{i}{k}\right)^{2} \leq \sum_{j} \max \left\{a_{t}(i, i)-a_{t}(i, j), 0\right\}+\frac{\tilde{n}_{t}(i / k)}{4 k^{2}} .
$$

Summing both sides of the last inequality and noting that $\sum_{i} \tilde{n}_{t}(i / k)=t$, we see that $t \tilde{C}(t) \leq R_{t}(F)+t /\left(4 k^{2}\right)$.

Sergiu $\mathrm{H}$ art (personal communication) has given a charming alternative proof, based on the minimax theorem, of the existence of a close to calibrated forecast. U nfortunately, $\mathrm{H}$ art's proof does not lead to an efficient algorithm for generating such a forecast. Fudenberg and L evine (1995) also give a (different) proof based on the minimax theorem. Their proof is longer than $\mathrm{H}$ arts' but has the virtue of leading to an efficient algorithm for finding a close-to-calibrated forecast. 


\section{NO EXTERNAL REGRET}

For any decision scheme $S$ let $L_{T}(S)$ be the (expected) total loss from using $S$ up to time $T .{ }^{6}$ Let $\mathscr{F}$ be a collection of different decision schemes. A decision scheme, $S$, is said to have no external regret with respect to $\mathscr{F}$ if

$$
L_{T}(S) \leq \min _{P \in \mathscr{F}} L_{T}(P)+o(T)
$$

for all sequences of states of the world. So, for large $T$, the time averaged loss from using $S$ is almost as good as the average loss of the best of the schemes in $\mathscr{F}^{?}$ ?

Given any finite set $\mathscr{F}$ of decision schemes, we show how to construct a decision scheme that has no external regret with respect to $\mathscr{F}$. Consider the case where $\mathscr{F}$ consists of just two schemes, $A$ and $B$. L et $L_{t}^{A}$ and $L_{t}^{B}$ be the loss incurred by using schemes $A$ and $B$, respectively, in time $t$. L et $C$ be a scheme that follows $A$ in time $t$ with probability $w_{t}$ and scheme $B$ with probability $1-w_{t}$. In effect, $C$ is a decision scheme whose decision set consists of just two options, do $A$ or do $B$. Then

$$
L_{T}(C)=\sum_{t=1}^{T}\left[w_{t} L_{t}^{A}+\left(1-w_{t}\right) L_{t}^{B}\right] .
$$

THEOREM 1. If $C$ is a no internal regret scheme, then $C$ has no external regret with respect to $\{A, B\}$.

Proof. Without loss of generality, we may assume that $L_{T}(A) \leq L_{T}(B)$. The regret associated with $C$ is

$$
R_{T}(C)=\max \left\{\sum_{t=1}^{T} w_{t}\left(L_{t}^{A}-L_{t}^{B}\right), 0\right\}+\max \left\{\sum_{t=1}^{T}\left(1-w_{t}\right)\left(L_{t}^{B}-L_{t}^{A}\right), 0\right\} .
$$

Since $R_{T}(C)=o(T)$, it follows that

$$
\max \left\{\sum_{t=1}^{T} w_{t}\left(L_{t}^{A}-L_{t}^{B}\right), 0\right\}+\max \left\{\sum_{t=1}^{T}\left(1-w_{t}\right)\left(L_{t}^{B}-L_{t}^{A}\right), 0\right\}=o(T) .
$$

Thus

$$
\max \left\{\sum_{t=1}^{T}\left(1-w_{t}\right)\left(L_{t}^{B}-L_{t}^{A}\right), 0\right\} \leq o(T)
$$

${ }^{6}$ The expectation is with respect to the randomization induced by $S$.

${ }^{7}$ Some authors, Littlestone and Warmuth (1994) and Feder et al. (1992), have studied the ratio $L_{T}(S) / \min _{P \in \mathscr{F}} L_{T}(P)$. H owever, bounds on the ratio can be derived from bounds on the difference $L_{T}(S)-\min _{P \in \mathscr{F}} L_{T}(P)$. 
Since $\max \{x, 0\} \geq x$, we deduce that

$$
\sum_{t=1}^{T}\left(1-w_{t}\right)\left(L_{t}^{B}-L_{t}^{A}\right) \leq o(T) .
$$

Adding $\sum_{t=1}^{T} w_{t} L_{t}^{A}$ to both sides of this last inequality, we obtain the required result.

Given that $C$ is a no internal regret forecast, we have from Section 2.2 that

$$
L_{T}(C)-\min \left\{L_{T}(A), L_{T}(B)\right\}=O(\sqrt{T}) .
$$

This bound is the best possible (Cesa-Bianchi et al., 1993). However, for particular loss functions or states of the world, it can be improved.

To extend the result to a set $\mathscr{F}$ of more than two decision schemes is easy. Start with two schemes, $A$ and $B \in \mathscr{F}$, and use the theorem to construct a scheme $Z^{0}$ that has no external regret with respect to the two of them. Now, take a third scheme $C$ in $\mathscr{F}$ and produce a scheme $Z^{1}$ with no external regret with respect to $Z^{0}$ and $C$. N otice that $Z^{1}$ is comparable to $\{A, B, C\}$. Continuing in this way we obtain

THEOREM 2. Given any finite set of decision schemes $\mathscr{F}$, there exists a (randomized) decision scheme $S$ with no external regret with respect to $\mathscr{F}$.

Interestingly, Theorem 2 has been proved many times in the last 40 years. A review of the titles of some of the papers that contain proofs of Theorem 2 (or special cases) explains why:

- Controlled Random Walks

- On Pseudo-games

- A R andomized Rule for Selecting Forecasts

- A pproximating the Bayes R isk in R epeated Plays

- A ggregating Strategies

- U niversal Portfolios

The first proof we are aware of is due to James Hannan (1957), where it arises in a game theoretic context. ${ }^{8}$

\footnotetext{
${ }^{8}$ We thank A Ido Rustichini for leading us to the paper by $\mathrm{H}$ annan. A las, it came to our attention only after we had reinvented the wheel in Foster and Vohra (1993).
} 


\subsection{An Application to Game Theory}

Consider a two-player game which will be played repeatedly, where the "loss" to the row player from playing strategy $i$ when the column player plays her strategy $j$ is $a_{i j}$. Suppose that the row player knows the proportion $y_{j}$ of times that the column player will play strategy $j$. Knowing this, the smallest (average) loss that the row player can receive is

$$
v(y)=\min _{i} \sum_{j} a_{i j} y_{j} .
$$

$\mathrm{H}$ annan (1957) showed that asymptotically, the row player can achieve $v(y)$ without knowing $y$ ahead of time, using randomization and the history of past plays. Call this the $\mathrm{H}$ annan theorem. Let us see how to derive it using Theorem $2 .^{9}$

O ur set of decision schemes, $\mathscr{F}$, will be the set of strategies that the row player has. The $i$ th scheme in $\mathscr{F}$ will be to choose the $i$ th strategy in each round. By Theorem 2 there is a scheme $S$ such that

$$
\min _{P \in \mathscr{F}} L_{T}(P) \leq L_{T}(S) \leq \min _{P \in \mathscr{F}} L_{T}(P)+o(T) .
$$

Dividing by $T$ and letting $T \rightarrow \infty$, we conclude that

$$
L_{T}(S) \rightarrow \frac{\min _{P \in \mathscr{F}} L_{T}(P)}{T} .
$$

However, $\left(\min _{P \in \mathscr{F}} L_{T}(P)\right) / T$ is just $v(y)$, where $y$ is the empirical average of the column players plays.

Notice that Theorem 2 does not require that the column player have a finite number of strategies or that the $a_{i j}^{\prime}$ be nonrandom. I nterestingly, Theorem 2 can be derived from Hannan's theorem itself. For a proof we refer the reader to Freund and Schapire (1996). For this reason we will sometimes refer to Theorem 2 as H annan's theorem.

It is also possible to derive Hannan's theorem using the existence of a close to calibrated forecast. The row player makes probability forecasts of the column player playing each of her strategies and then plays a best response. If the forecast is close to calibrated, row time-averaged payoffs converge to $v(y)$. This proof requires that the row player know all of the column player's strategies.

B efore continuing with our history of Theorem 2, we mention one interesting consequence of it for zero-sum games. In this case $a_{i j}$ is the loss to

\footnotetext{
${ }^{9} \mathrm{H}$ annan's proof required that the row player know the entire game matrix ahead of time. By relying on Theorem 2, we shall see that this is not necessary. It is enough for the player to know the column of the matrix corresponding to the strategy played by column.
} 
the row player and the gain to the column player. L et $v$ be the value of this zero-sum game. By the easy part of the minimax theorem,

$$
\frac{L_{T}(S)}{T} \geq v \geq v(y) \text {. }
$$

Since $S$ has no external regret with respect to $\mathscr{F}$, it follows that

$$
\frac{L_{T}(S)}{T}-v \rightarrow 0
$$

as $T \rightarrow \infty$. The actual rate of convergence (first established by $\mathrm{H}$ annan) is $\frac{1}{\sqrt{T}}$, and this is the best possible (see Cesa-Bianchi et al., 1993). Thus, any algorithm for constructing a no-external-regret scheme is an algorithm for finding the value of a zero-sum game, and so for solving a linear program. For a detailed treatment see Freund and Schapire (1996). ${ }^{10}$

A short time after Hannan announced his result, David Blackwell (1956a), showed how Hannan's theorem could be obtained as a corollary of his approachability theorem (Blackwell, 1956b). To use the theorem one needs to define an auxiliary game with vector-valued payoffs and a target set. If the row player chooses strategy $i$ and the column player chooses strategy $j$, the payoff is an $(n+1)$-vector with a 1 in the $j$ th position, $a_{i j}$ in the $(n+1)$ st position, and zeros everywhere else. Here $n$ is the number of strategies of the column player. The target set $G$ is the set of vectors $y$ in $\Re^{n+1}$ such that

1. $\sum_{j=1}^{n} y_{j}=1$.

2. $y_{n+1} \leq \sum_{j=1}^{n} a_{i j} y_{j}$ for all $i$.

3. $y_{j} \geq 0$ for $1 \leq j \leq n$.

If $y$ is the vector that represents the proportion of times the column player has played each of his or her strategies, then the vector $(y, v(y))$ is in the target set $G$. So, to prove Hannan's theorem, it is sufficient to show that this target set is approachable.

Independently but 9 years later in 1968, Banos (1968) also derived $\mathrm{H}$ annan's theorem. The proof given is quite complicated but proves it for the case where the payoffs are random variables and the row player knows only his or her own pure strategies. A decade after that, M egiddo (1980) also proposed and proved Hannan's theorem, this time 23 years after the original. It is clear from the comments in that paper that Megiddo became aware of the paper by Banos after his own paper was in press. M egiddo's

\footnotetext{
${ }^{10}$ It is interesting to note that for non-zero-sum games, if players use a no-internal-regret scheme, one gets convergence to the correlated equilibrium of the game. In the case of zerosum games, the sets of correlated equilibria and $\mathrm{N}$ ash equilibria coincide. The details may be found in Foster and Vohra (1997) or H art and M as-Colell (1996).
} 
proof is simpler than Banos' but is still quite elaborate when compared with the arguments given here.

In 1994, the theorem was (re)discovered again by Fudenberg and L evine (1999a). The proof given is different from the ones given by $\mathrm{H}$ annan, Blackwell, $\mathrm{B}$ anos, and $\mathrm{M}$ egiddo. In their scheme strategies are played in proportion to their payoffs with exponential weights. This, as we explain later, has been the most popular method for proving $\mathrm{H}$ annan's theorem. ${ }^{11}$ I $\mathrm{n}$ a sequel to their 1994 paper, Fudenberg and Levine (1999b) investigate a generalization of Hannan's theorem. Instead of asking if the player could do as well as if she knew the frequency of outcomes in advance, we could divide the samples into subsamples and ask if the player could do as well as if she knew the frequencies of the subsamples and was told in advance which subsample the observation was going to be drawn from. They give a positive result, using a variation of the regret idea introduced in the previous section.

The most recent (re)discovery of $\mathrm{H}$ annan's theorem in a game theory context we are aware of is the paper by Auer et al. (1995). This last paper is of interest because it provides other applications of Theorem 2 as well as some refinements. In particular, it extends Hannan's theorem to the case where the row player knows only the payoff from the strategy played in each round, thus providing for an on-line version of the classical bandit problem. ${ }^{12}$

\subsection{An Application to Sequence Prediction}

A problem that has received a great deal of attention in the computer science literature is that of predicting a sequence of 0's and 1's with "few" mistakes. The problem has stimulated a number of proofs of special cases of Theorem 2. All have involved the use of an algorithm that chooses to predict 0 or 1 in proportion to their payoffs with exponential weights. The exponential-weighted algorithm just alluded to was introduced by Littlestone and Warmuth (1989), DeSantis et al. (1992), Feder et al. (1992), and Vovk (1990) at about the same time. Vovk (1990) shows how the exponential-weighted algorithm can be used to prove Theorem 2 for any bounded loss function (but the states of the world are either 0 or 1 ).

Cesa-Bianchi, Freund, Helmbold, Haussler, Schapire, and Warmuth (Cesa-Bianchi et al., 1993) study the special case of the absolute loss

\footnotetext{
${ }^{11}$ We note that the important ingredients for a proof of $\mathrm{H}$ annan's theorem can also be found in Easley and Rustichini (1995). That paper does not contain an explicit statement of the theorem or proof.

${ }^{12} \mathrm{~A}$ similar result can be found in Foster and Vohra (1993).
} 
function, ${ }^{13}$ establishing the best possible rates of convergence under various partial information scenarios as a function of $T$ and the number of schemes in $\mathscr{F}$. For example, the decision maker knows an upper bound on the total loss of the best scheme in $\mathscr{F}$ or knows the length of the game, $T$ (see also Haussler et al. (1995)).

In the case where the state of the world in each period is not binary, Littlestone and Warmuth (1989) and Kivinen and Warmuth (1993) show that Theorem 2 holds, but only for a particular loss function. Within this literature, Theorem 2 as we have stated it was obtained by Chung (1994) and Freund and Schapire (1995).

We close this section with a pleasing implication of Theorem $2 .{ }^{14}$ In any sequence of 0 's and 1's let $u_{t}$ be the fraction of 1 's that have appeared up to time $t$. Suppose you have been predicting the next element of the sequence. Let $f_{t}$ be the expected fraction of incorrect predictions you have made up to time $t$.

THEOREM 3. For any sequence of 0 's and 1's there is a way to predict the next element in the sequence so that

$$
f_{t} \rightarrow \min \left\{u_{t}, 1-u_{t}\right\}
$$

as $t \rightarrow \infty$.

Proof. Define the loss function $L_{t}$ at time $t$ to take the value 1 if an incorrect prediction has been made and 0 otherwise. Let $A$ be the decision/prediction scheme that predicts a 1 at each time and let $B$ be the scheme that predicts a 0 every time. Clearly, $L_{t}(A) / t=1-u_{t}$ and $L_{t}(B) / t=u_{t}$. By Theorem 2 there is a scheme $C$ such that

$$
L_{t}(C) \leq \min \left\{L_{t}(A), L_{t}(B)\right\}+O(t) .
$$

Divide through by $t$ and the theorem is proved.

Thus, the fraction of incorrect predictions will never exceed one-half and could be lower if there were a bias in the sequence toward 0's or 1's.

\subsection{Statistics}

Within statistics Foster (1991) proves a version of Theorem 2 for the case of a quadratic loss function and two possible states of the world. A paper by Foster and Vohra (1993) contains Theorem 2 in the form stated

\footnotetext{
${ }^{13}$ The loss at time $t$ is $\left|p_{t}-X_{T}\right|$, where the $p_{t}$ is the prediction at time $t$ and $X_{t}=0,1$ is the state of the world.

${ }^{14}$ We believe this was first observed by David Blackwell.
} 
here. The proof is motivated by statistical considerations which we outline here.

O ne can view the average losses accumulated thus far by the two schemes $A$ and $B$ as sample means. Presumably the sample means should tell one something about the true mean. So the question becomes this: when is the difference in sample means sufficiently large for us to conclude that scheme $A$ (or $B$ ) should be the one to follow on the next round? $U$ sually such a question is answered by examining how many standard deviations one sample mean is from the other. In our case, we can make no appeal to the central limit theorem to posit a distribution and so compute a standard deviation. Even so, let us suppose that the losses incurred by each scheme on each round are independent random variables. Since the losses are bounded above by 1 , we would expect the difference in the average loss of the two schemes after $T$ rounds to be $O(1 / T)$ and the standard deviation of that difference to be $O(1 / \sqrt{T})$.

If the difference in the average losses of the two schemes were less than $O(1 / \sqrt{T})$, we would conclude that there was no difference between the two schemes and so randomly select which scheme to follow on the next round.

If the difference in the average losses of the two schemes exceeded $O(1 / \sqrt{T})$, we would conclude that one scheme was superior to the other and use it on the next round.

This is essentially the scheme proposed by Foster and Vohra (1998). In the case where the difference in the average losses of the two schemes is less than $O(1 / \sqrt{T})$, one randomizes over the two schemes with probability $(1 / 2-\epsilon, 1 / 2+\epsilon)$, where $\epsilon$ is a small number that depends on the average difference of the accumulated losses thus far.

\subsection{An Application to Finance}

In this section we show how Theorem 2 can be used to obtain a result first derived by Cover (1991) by other means. Along the way we will describe a trick for generalizing Theorem 2, under certain conditions, to the case where $\mathscr{F}$ consists of a continuum of decision schemes.

I magine a financial world consisting of just two stocks, $A$ and $B$. Let $A_{t}$ and $B_{t}$ be the value of stocks $A$ and $B$, respectively, at time $t$. We assume that $A_{t}$ and $B_{t}$ are bounded. To avoid extra notation suppose that $A_{0}=B_{0}=1$ and that our initial wealth is 1 as well. The return on stock $A$ at time $t$ will be $A_{t} / A_{t-1}$. So the growth rate at time $t$ of stock $A$ will be $\ln \left(A_{t} / A_{t-1}\right)$. Since

$$
A_{t}=\prod_{r=1}^{t} \frac{A_{r}}{A_{r-1}}
$$


it follows that $\left(\ln A_{t}\right) / t$ will be the average growth rate of stock $A$ over $t$ periods. ${ }^{15}$ We will use Theorem 2 (with inequalities reversed to account for gains rather than losses) with $\mathscr{F}$ consisting of the following two schemes: buy and hold stock $A$ only and buy and hold stock $B$. Interpret the probability of choosing each of these schemes as the proportion of our current wealth that should be invested in each stock. In particular, if $w_{t}$ is the "probability" of picking stock $A$ at time $t$, the growth rate at time $t$ will be $w_{t} \ln \left(A_{t} / A_{t-1}\right)+\left(1-w_{t}\right) \ln \left(B_{t} / B_{t-1}\right)$. Given this, we can construct a changing portfolio of the two stocks, $C$, say, whose value at time $t, C_{t}$, satisfies

$$
\frac{\ln C_{t}}{t} \geq \max \left\{\frac{\ln A_{t}}{t}, \frac{\ln B_{t}}{t}\right\}-O\left(\frac{1}{\sqrt{t}}\right) .
$$

In effect, the average growth rate of $C$ is asymptotically equal to the better of the growth rates of $A$ and $B{ }^{16}$ It is not hard to see that this result holds for any finite number of stocks.

The previous result shows only that we can, without advance knowledge of the future, match the average growth rate of the best stock. Could we, without being clairvoyant, match the growth rate of the best portfolio of the two stocks? ${ }^{17}$ The answer is a qualified yes. We can match the growth rate of the best portfolio from the class of constant portfolios. Such portfolios maintain a constant proportion of their wealth in each stock. For example, in each period maintain one-third of the value of the portfolio in $A$ and the remainder in $B$. Such a portfolio needs to be adjusted from one period to the next to maintain this fraction.

A s there are as many constant portfolios as numbers in the interval $[0,1]$, a direct application of Theorem 2 is not possible. The trick is to pick a finite collection of constant portfolios that "cover" the set of all constant portfolios. If the collection is large enough, one can guarantee that one of those portfolios has a growth rate close to the average growth rate of the best constant portfolio.

Each constant portfolio can be represented by a single number in the interval $[0,1]$. That number is the fraction of the portfolio's wealth invested in stock $A$. Let $V_{t}(x)$ be the value of the constant portfolio $x$ at time $t$. Pick an integer $k$ (exact value to be specified later), and let $\mathscr{F}$ be the set of constant portfolios $\{1 / k, 2 / k, \ldots,(k-1) / k\}$. A pplying Theorem 2, we deduce the existence of investment scheme $C$ with value $C_{t}$ at time $t$

\footnotetext{
${ }^{15}$ In finance this is called the internal rate of return.

${ }^{16}$ In this special case, the $1 / \sqrt{t}$ term can be improved to $1 / t$.

${ }^{17}$ The portfolio is assumed to have the same same starting wealth as we do.
} 
such that

$$
\frac{\ln C_{t}}{t} \geq \max _{x \in \mathscr{F}} \ln V_{t}(x)-\left(\frac{1}{\sqrt{t}}\right) .
$$

Let $z$ be the constant portfolio in $\mathscr{F}$ with the largest value and let $y$ be the constant portfolio with the largest value overall, i.e.,

$$
V_{t}(y)=\max _{x \in[0,1]} V_{t}(x)
$$

We show that the difference between $\left(\ln V_{t}(z)\right) / t$ and $\left(\ln V_{t}(y)\right) / t$ is small.

For any $x \in[0,1]$,

$$
V_{t}(x)=\prod_{j=0}^{t}\left[x A_{j}+(1-x) B_{j}\right]=\prod_{j=0}^{t}\left[B_{j}+x\left(A_{j}-B_{j}\right)\right] .
$$

Hence,

$$
\ln V_{t}(x)=\sum_{j=0}^{t} \ln \left(B_{j}+x\left(A_{j}-B_{j}\right)\right) .
$$

Choose $\frac{r}{k}$ closest to $y$. Then $\left|y-\frac{r}{k}\right| \leq \frac{1}{k}$. Now,

$$
\ln V_{t}(y)-\ln V_{t}(z) \leq \ln V_{t}(y)-\ln V_{t}(r / k) .
$$

The right-hand side of this last inequality is just

$$
\sum_{j=0}^{t}\left[\ln \left(B_{j}+y\left(A_{j}-B_{j}\right)\right)-\ln \left(B_{j}+(r / k)\left(A_{j}-B_{j}\right)\right] .\right.
$$

Each term of the sum is

$$
\ln \frac{B_{j}+y\left(A_{j}-B_{j}\right)}{B_{j}+(r / k)\left(A_{j}-B_{j}\right)}=\ln \frac{1+y\left(A_{j}-B_{j}\right) / B_{j}}{1+(r / k)\left(A_{j}-B_{j}\right) / B_{j}} .
$$

Suppose $A_{j}-B_{j} \geq 0$, the argument is similar for the converse. From the choice of $r, y \leq \frac{r+1}{k}$. So

$$
1+\frac{y\left(A_{j}-B_{j}\right)}{B_{j}} \leq 1+\frac{(r+1)\left(A_{j}-B_{j}\right)}{k B_{j}} .
$$

Hence

$$
\ln \frac{1+y\left(A_{j}-B_{j}\right) / B_{j}}{1+(r / k)\left(A_{j}-B_{j}\right) / B_{j}} \leq \ln (1+O(1 / k)) \leq O(1 / k) .
$$

Therefore

$$
\begin{aligned}
\ln V_{t}(y)-\ln V_{t}(z) & \leq \sum_{j=0}^{t}\left[\ln \left(B_{j}+y\left(A_{j}-B_{j}\right)\right)-\ln \left(B_{j}+\frac{r\left(A_{j}-B_{j}\right)}{k}\right)\right] \\
& \leq O(t / k) .
\end{aligned}
$$


So, $\left(\ln V_{t}(y)\right) / t-\left(\ln V_{t}(z)\right) / t \leq O(1 / k)$. Thus, given any $\epsilon>0$, we can choose $k$ and $t$ sufficiently large so that

$$
\frac{\ln C_{t}}{t} \geq \frac{\ln V_{t}(y)}{t}-\epsilon .
$$

A gain this argument is easily generalized to the case of more than two stocks. ${ }^{18}$

The main idea used in extending Theorem 2 to a continuum of schemes is that the loss function be "smooth." Suppose we can associate with each scheme $\mathscr{F}$ a point $x$ in a compact set with metric $\rho$, say. Let $L_{t}(x)$ be the loss from using scheme $x$ at time $t$. If $\left|L_{t}(x)-L_{t}(y)\right| \leq O(\rho(x, y))$ for all points $x$ and $y$, then, by covering $\mathscr{F}$ with a sufficiently fine grid of points, we can mimic the argument above to show that Theorem 2 holds.

\subsection{The Exponential Weighted Algorithm}

$M$ any of the proofs of Theorem 2 have involved the use of an algorithm that selects a decision in proportion to its loss with exponential weights. In this section we suggest why this is a natural way way to prove Theorem 2.

R eturn again to the world of two stocks. Theorem 2 implied the existence of a portfolio $C$ whose value at time $t$ satisfied

$$
\frac{\ln C_{t}}{t} \geq \max \left\{\frac{\ln A_{t}}{t}, \frac{\ln B_{t}}{t}\right\}-O\left(\frac{1}{\sqrt{t}}\right) .
$$

The portfolio that does this is the one that divides the current wealth between the two stocks in proportion to the values of the individual stocks. Thus at time $t$, a fraction

$$
w_{t}=\frac{A_{t-1}}{A_{t-1}+B_{t-1}}
$$

of current wealth is invested in stock $A$. To see why this works, consider what happens at $t=0$. Since $A_{0}=B_{0}=1$ and initial wealth is 1 , this portfolio invests $\$ 1 / 2$ in $A$ and $\$ 1 / 2$ in $B$. At time $t=1$ this portfolio has value $\left(A_{1}+B_{1}\right) / 2$. The portfolio now invests

$$
\frac{A_{1}}{A_{1}+B_{1}}\left(\frac{A_{1}+B_{1}}{2}\right)=\frac{A_{1}}{2}
$$

in stock $A$ and the remainder, $B_{1} / 2$, in stock $B$. So, at time $t=2$, the value of the portfolio will be $\left(A_{2}+B_{2}\right) / 2$. Continuing in this fashion, it is easy to see that

$$
C_{t}=\frac{A_{t}+B_{t}}{2}
$$

${ }^{18}$ The dependence on $k$ can be removed using a standard argument. 
Now, from the properties of the logarithm function, we deduce that

$$
\ln C_{t}=\ln \left(\frac{A_{t}+B_{t}}{2}\right) \geq \max \left\{\ln A_{t}, \ln B_{t}\right\}-\ln 2 .
$$

Dividing by $t$, we obtain the required result. ${ }^{19}$

Now let us consider the more general setting. We have two schemes, $A$ and $B$. The gains at time $t$ from using schemes $A$ and $B$ are $G_{t}^{A}$ and $G_{t}^{B}$, respectively. ${ }^{20}$ A ssume that $G_{0}^{A}=0=G_{0}^{B}$ and all gains are bounded above by 1 . The goal is to construct a scheme $C$ such that

$$
\sum_{t=0}^{T} G_{t}^{C} \geq \max \left\{\sum_{t=0}^{T} G_{t}^{A}, \sum_{t=0}^{T} G_{t}^{B}\right\}-o(T) .
$$

To do this we associate with scheme $A$ a stock $A^{\prime}$ whose value $A_{t}^{\prime}$ at time $t$ is $\prod_{t=0}^{T} x^{G_{t}^{A}}$ (similarly with scheme $B$ ). The number $x>1$ will be chosen later. The advantage of this construction is that

$$
\ln A_{T}^{\prime}=\ln x \sum_{t=0}^{T} G_{t}^{A}
$$

and

$$
\ln B_{T}^{\prime}=\ln x \sum_{t=0}^{T} G_{t}^{B}
$$

U sing the previous argument, we construct a portfolio, $C^{\prime}$, that invests a fraction

$$
w_{T}=\frac{x^{\sum_{t=0}^{T-1} G_{t}^{A}}}{x^{\sum_{t=0}^{T-1} G_{t}^{A}}+x^{\sum_{t=0}^{T-1} G_{t}^{B}}}
$$

of the wealth at time $T$ in stock $A^{\prime}$. Hence

$$
\ln C_{T}^{\prime} \geq \ln x \max \left\{\sum_{t=0}^{T} G_{t}^{A}, \sum_{t=0}^{T} G_{t}^{B}\right\}-o(T) .
$$

Let $C$ be the scheme that chooses scheme $A$ at time $t$ with probability $w_{t}$. The trick now is to use what we know about $\ln C_{t}^{\prime}$ to prove that $C$ has no external regret with respect to $A$ and $B$.

\footnotetext{
${ }^{19} \mathrm{~N}$ otice that we get the $1 / t$ term rather than $1 / \sqrt{t}$.

${ }^{20} \mathrm{We}$ focus on gains rather than losses. The reason will become clearer later.
} 
Let $a_{t}=\sum_{j=1}^{t} G_{j}^{A}$ and $b_{t}=\sum_{j=1}^{t} G_{j}^{B}$. Then

$$
\begin{aligned}
w_{t} & =\frac{x^{a_{t-1}}}{x^{a_{t-1}}+x^{a_{t-1}}}, \\
x^{a_{t}} & =x^{a_{t-1}} x^{G_{t}^{A}} \leq x^{a_{t-1}}\left(1+(x-1) G_{t}^{A}\right)
\end{aligned}
$$

and

$$
x^{b_{t}}=x^{b_{t-1}} x^{G_{t}^{B}} \leq x^{b_{t-1}}\left(1+(x-1) G_{t}^{B}\right) .
$$

Hence

$$
x^{a_{t}}+x^{b_{t}} \leq\left(x^{a_{t-1}}+x^{b_{t-1}}\right)\left[1+(x-1)\left(w_{t} G_{t}^{A}+\left(1-w_{t}\right) G_{t}^{B}\right)\right] .
$$

$\mathrm{U}$ sing the fact that $1+y \leq e^{y}$, we deduce that

$$
x^{a_{t}}+x^{b_{t}} \leq\left(x^{a_{t-1}}+x^{b_{t-1}}\right) e^{(x-1)\left(w_{t} G_{t}^{A}+\left(1-w_{t}\right) G_{t}^{B}\right)} .
$$

$\mathrm{U}$ sing this last inequality recursively, we obtain

$$
x^{a_{t}}+x^{b_{t}} \leq\left(x^{a_{0}}+x^{b_{0}}\right) \prod_{j=1}^{t} e^{(x-1)\left(w_{j} G_{j}^{A}+\left(1-w_{j}\right) G_{j}^{B}\right)} .
$$

Since $a_{0}=0=b_{0}$, we get

$$
x^{a_{t}}+x^{b_{t}} \leq 2 \prod_{j=1}^{t} e^{(x-1)\left(w_{j} G_{j}^{A}+\left(1-w_{j}\right) G_{j}^{B}\right]} .
$$

Taking logs and noting that

we get

$$
C_{t}^{\prime}=\frac{x^{a_{t}}+x^{b_{t}}}{2}
$$

$$
(x-1) \sum_{j=1}^{t}\left(w_{j} G_{j}^{A}+\left(1-w_{j}\right) G_{j}^{B}\right) \geq \ln C_{t}^{\prime} .
$$

U sing what we know about $C_{t}^{\prime}$, we derive

$$
\sum_{j=1}^{T}\left(w_{j} G_{j}^{A}+\left(1-w_{j}\right) G_{j}^{B}\right) \geq \frac{\ln x}{x-1} \max \left\{\sum_{t=0}^{T} G_{t}^{A}, \sum_{t=0}^{T} G_{t}^{B}\right\}-o(T) .
$$

The left-hand side of the above is the expected gain from using scheme $C$ up to time $T$. If we choose $x=1+\frac{1}{\sqrt{T}}$ and use the fact that the maximum gain in any period is 1 , we conclude that

$$
\sum_{j=1}^{t}\left(w_{j} G_{j}^{A}+\left(1-w_{j}\right) G_{j}^{B}\right) \geq \max \left\{\sum_{t=0}^{T} G_{t}^{A}, \sum_{t=0}^{T} G_{t}^{B}\right\}-o(T) .
$$

There is one drawback to the exponential-weighted majority algorithm. It relies on a parameter, $x$, that depends on $T$. Thus, one must know ahead of time how many periods the decision problem must run. 


\section{APPENDIX: APPROACHABILITY THEOREM}

R ow $(\mathrm{R})$ and Column (C) repeatedly meet to play an $m \times n$ matrix game. If $\mathrm{R}$ chooses her strategy $i$ and $\mathrm{C}$ chooses his strategy $j$, the payoff is a vector $v_{i j}$ in some compact space. ${ }^{21}$ Let $i_{t}$ and $j_{t}$ be the strategies chosen by $\mathrm{R}$ and $\mathrm{C}$, respectively, in round $t$. Both $\mathrm{R}$ and $\mathrm{C}$ are concerned with the long-term average of the payoffs:

$$
A_{T}=\sum_{t=1}^{T} v_{i_{t} j_{t}} / T
$$

In the space in which the vector payoffs reside there is a convex set $G$, called the target set. R's goal is to play the game so as to force $A_{T}$, no matter what the starting point, to approach $G$ arbitrarily closely almost surely, i.e., $d\left(A_{T}, G\right) \rightarrow 0$ almost surely as $T \rightarrow \infty$. If $\mathrm{R}$ can succeed at approaching $G$, the set $G$ is said to be approachable.

In the case when $G$ is a convex set, Blackwell (1956) gave a necessary and sufficient condition for a convex target set to be approachable. B efore giving a formal statement and proof of the result, we describe the intuition behind it. Consider first the simpler case when the target set $G$ is a halfspace, say $\{x: a \bullet x \leq 0\}$. Suppose $\mathrm{R}$ has a mixed strategy over her rows, $\lambda$, so that no matter what $C$ does, the outcome in expectation is a vector in the half-space or on the defining hyperplane. That is,

$$
a \bullet \sum_{i=1}^{m} \lambda_{i} v_{i j} \leq 0
$$

for all $j=1, \ldots, n$. Notice that if $\mathrm{R}$ does not have such a strategy, then the half-space is clearly not approachable. So this requirement is necessary for approachability. Now we outline why it is sufficient.

Suppose $\mathrm{R}$ plays this mixed strategy $\lambda$ in every round. Then, in each round, the outcome $v_{i_{t} j_{t}}$ is a random vector with the property that $E(a \bullet$ $\left.v_{i_{t} j_{t}}\right) \leq 0$. Since

$$
A_{T}=\sum_{t=1}^{T} v_{i_{t} j_{t}} / T,
$$

it follows that

$$
a \bullet A_{T}=\sum_{t=1}^{T}\left(a \bullet v_{i_{t} j_{t}}\right) / T \text {. }
$$

\footnotetext{
${ }^{21} \mathrm{M}$ ore generally, the payoff can be a vector drawn from a distribution that depends on $i$ and $j$. The proof described here easily extends to this case.
} 
Thus, $a \bullet A_{T}$ is the average of a collection of random variables, each of which is nonpositive in expectation. $O$ ne is tempted to now invoke the law of large numbers to conclude that $a \bullet A_{T}$ must be negative almost surely. The catch is that the variables $a \bullet v_{i_{t} j_{t}}$ may not be independent of each other. They are determined not just by R's choices but by $C$ 's as well. A nd $C$ may be playing some elaborate strategy that depends on the outcome of previous rounds of the game. H owever, by using the appropriate law of large numbers result that accounts for dependencies, this difficulty can be overcome. Thus approachability of a half-space reduces to deciding whether a collection of linear inequalities is satisfied.

N ow suppose the target set is not necessarily a half-space. Suppose $A_{T} \notin$ $G$ to be the current average payoff and $g$ the point in $G$ closest to $A_{T}$. L et $l$ be the plane perpendicular to the line joining $A_{T}$ and $g$ that touches $G$. Such a plane can be found by virtue of the separating hyperplane theorem. Suppose that $C$ has a strategy (possibly mixed) such that no matter what pure strategy $\mathrm{R}$ plays, the outcome is a vector $v$ on the same side of $l$ as $A_{T}$. That is, the half-space defined by $l$ containing $G$ is not approachable. In this case $G$ is not approachable. Thus, a necessary condition for approachability is that each tangent hyperplane of $G$ is approachable. Now we outline why this is sufficient. To see why such a conclusion is plausible, assume that $T$, the number of rounds played so far, is very large. Let $v$ be the payoff on the "right" side of $l$ that $\mathrm{R}$ can force in round $T+1$. Then, the average payoff becomes $\frac{T}{T+1} A_{T}+v /(T+1)$. Since $v$ is on the other side of $l$ from $A_{T}$, it is not hard to see that the new average, $\frac{T}{T+1} A_{T}+v /(T+1)$, is a little closer to $G$ than $A_{T}$ was. Notice that the compactness requirement is used here to guarantee that for any $v$, there is a $T$ sufficiently large so that $v /(T+1)$ will be small enough.

THEOREM 4. A convex set $G$ is approachable if and only if every tangent hyperplane of $G$ is approachable.

Blackwell's theorem has been generalized in two ways that are useful. First, in Vielle (1992) a result for nonconvex target sets is established by weakening appropriately the notion of approachability. In (Lehrer, 1997), Blackwell's theorem is extended to the case of infinite-dimensional spaces. We do not consider these generalizations here.

\subsection{Proof of Blackwell's Approachability Theorem}

Suppose, then, that $\mathrm{R}$ can force the outcome of the next round of play to be, in expectation, on the same side of $l$ as $G$. We will show that the set $G$ is approachable. Let $D$ be the largest distance between any two points 
in the set of possible payoffs. ${ }^{22}$ Let $d\left(A_{t}, G\right)$ be the distance from the current average $A_{t}$ to the nearest point in $G$. Our goal is to show that $d\left(A_{T}, G\right)$ goes to zero almost surely as $T \rightarrow \infty$. We do this by estimating $P\left(d\left(A_{T}, G\right) \geq \delta\right)$ from above.

Let $M_{T}=T^{2} d\left(A_{T}, G\right)^{2}-2 T D^{2}$. We prove two things about $M_{T}$ : first, that it is a super-martingale, i.e., $E^{T}\left(M_{T+1}\right) \leq M_{T}$; second, that $\mid M_{T+1}-$ $M_{T} \mid \leq(6 T+3) D^{2}$. From these two facts we will show that $d\left(A_{T}, G\right)$ converges almost surely to zero.

LEMMA 1. $M_{T}$ is a super-martingale.

Proof. Let $c_{T}$ be the closest point to $A_{T}$ in the set $G$. Then

$$
d\left(A_{T+1}, G\right) \leq d\left(A_{T+1}, c_{T}\right) .
$$

By our assumption that $\mathrm{R}$ has a strategy $w_{i}$ to follow, we know that for all $j$,

$$
\left(\sum_{i} w_{i}^{T+1} v_{i, j_{T+1}}-c_{T}\right)^{\prime}\left(A_{T}-c_{T}\right) \leq 0 .
$$

Let $a_{T+1}=\sum_{i} w_{i} v_{i, j}$. Thus,

$$
\begin{aligned}
d\left(A_{T+1}, c_{T}\right)^{2}= & \left(A_{T+1}-c_{T}\right)^{2} \\
= & \left(\frac{T}{T+1} A_{T}+\frac{1}{T} a_{T+1}-c_{T}\right)^{2} \\
= & \left(\frac{T}{T+1} A_{T}-\frac{T}{T+1} c_{T}\right)^{2} \\
& +2\left(\frac{T}{T+1} A_{T}-\frac{T}{T+1} c_{T}\right)^{\prime} \\
& \times\left(\frac{1}{T}\left(a_{T+1}-c_{T}\right)+\left(\frac{1}{T}\left(a_{T+1}-c_{T}\right)\right)^{2}\right. \\
\leq & \left(\frac{T}{T+1}\right)^{2} d\left(A_{T}, c_{T}\right)+\left(\frac{1}{T}\left(a_{T+1}-c_{T}\right)\right)^{2} \\
\leq & \left(\frac{T}{T+1}\right)^{2} d\left(A_{T}, G\right)+\frac{D^{2}}{T^{2}} .
\end{aligned}
$$

Thus,

$$
(T+1)^{2} d\left(A_{T+1}, G\right)^{2} \leq T^{2} d\left(A_{T}, G\right)^{2}+\left(\frac{T+1}{T}\right)^{2} D^{2} .
$$

${ }^{22}$ This is finite by compactness. 
Bounding $\frac{T+1}{T}$ by the crude bound of 2 , we get

$$
(T+1)^{2} d\left(A_{T+1}, G\right)^{2} \leq T^{2} d\left(A_{T}, G\right)^{2}+4 D^{2} .
$$

Writing this in terms of $M_{T}$, we get

$$
\begin{aligned}
E^{T}\left(M_{T+1}\right) & =E^{T}\left((T+1)^{2} d\left(L_{T+1}, G\right)^{2}-4(T+1) D^{2}\right) \\
& \leq E^{T}\left(T^{2} d\left(L_{T}, G\right)^{2}+4 D^{2}-4(T+1) D^{2}\right) \\
& =E^{T}\left(T^{2} d\left(L_{T}, G\right)^{2}-4 T D^{2}\right) \\
& =E^{T}\left(M_{T}\right) \\
& =M_{T} .
\end{aligned}
$$

LEMMA 2. $\left|M_{T+1}-M_{T}\right| \leq(6 T+3) D^{2}$.

Proof. Note that $\left|A_{T+1}-A_{T}\right| \leq D / T$. By convexity the closest point in $G$ to $A_{T+1}$ is no more than distance $D / T$ from the closest point in $G$ to $A_{T}$, i.e., $\left|c_{T+1}-c_{T}\right| \leq D / T$. By using the triangle inequality twice we see that $\left|d\left(A_{T+1}, G\right)-d\left(A_{T}, G\right)\right| \leq 2 D / T$. H ence,

$$
\begin{aligned}
M_{T+1}-M_{T}= & (2 T+1) d\left(A_{T+1}, G\right)^{2}+T^{2}\left(d\left(A_{T+1}, G\right)-d\left(A_{T}, G\right)\right) \\
& \times\left(d\left(A_{T+1}, G\right)+d\left(A_{T}, G\right)\right)-2 D^{2} .
\end{aligned}
$$

Thus,

$$
\left|M_{T+1}-M_{T}\right| \leq(2 T+1) D^{2}+4 T^{2} D^{2} / T+2 D^{2}=(6 T+3) D^{2} .
$$

Lemma 3. $d\left(A_{t}, G\right) \rightarrow 0$ almost surely as $T \rightarrow \infty$.

Proof. Let $S_{t}=M_{T} /(6 T+3) D^{2}=\sum_{t=1}^{T} X_{t}$, where each

$$
X_{t}=\frac{M_{t}-M_{t-1}}{(6 T+3) D^{2}}
$$

has an expectation less than zero and $\left|X_{t}\right| \leq \frac{6 t+3}{6 T+3} \leq 1$. We now want to show that $P\left(M_{T} \geq \epsilon T\right)$ goes exponentially fast to zero.

First note that

$$
e^{y} \leq 1+y+y^{2}
$$

if $y \leq 1$. So,

$$
E^{t-1}\left(e^{\alpha X_{t}}\right) \leq 1+\alpha E^{t-1}\left(X_{t}\right)+\alpha^{2} E^{t-1}\left(\left(X_{t}\right)^{2}\right)
$$


if $\alpha \leq 1$. Plugging in what we know about $X_{t}$,

$$
E^{t-1}\left(e^{\alpha X_{t}}\right) \leq 1+\alpha^{2} .
$$

Now,

$$
\begin{aligned}
P\left(S_{T} \geq \epsilon T\right) & =P\left(e^{\alpha S_{T}} \geq e^{\alpha \epsilon T}\right) \\
& \leq \frac{E\left(e^{\alpha S_{T}}\right)}{e^{\alpha \epsilon T}} \\
& =\frac{E\left(\prod_{t=1}^{T} e^{\alpha X_{t}}\right)}{e^{\alpha \epsilon T}} \\
& =\frac{\prod_{t=1}^{T} E^{t-1}\left(e^{\alpha X_{t}}\right)}{e^{\alpha \epsilon T}} \\
& \leq \frac{\prod_{t=1}^{T}\left(1+\alpha^{2}\right)}{e^{\alpha \epsilon T}} \\
& \leq \frac{\left(1+\alpha^{2}\right)^{T}}{e^{\alpha \epsilon T}} \\
& \leq \frac{e^{\alpha^{2} T}}{e^{\alpha \epsilon T}} \\
& =e^{\alpha(\alpha-\epsilon) T} .
\end{aligned}
$$

If we take $\alpha=\epsilon / 2$, then

$$
P\left(M_{T} \geq \epsilon T(6 T+3) D^{2}\right)=P\left(S_{T} \geq \epsilon T\right) \leq e^{-\epsilon^{2} T / 2} .
$$

Now substituting in the definition of $M_{T}$, we get

$$
P\left[T^{2} d\left(A_{t}, G\right)^{2}-2 T D^{2} \geq \epsilon T(6 T+3) D^{2}\right] \leq e^{-\epsilon^{2} T / 2},
$$

which solves to

$$
P\left[d\left(A_{t}, G\right)^{2} \geq 2 D^{2} / T+\epsilon(6+3 / T) D^{2}\right] \leq e^{-\epsilon^{2} T / 2} .
$$

For sufficiently large $T, 2 D^{2} / T+\epsilon(6+3 / T) D^{2}<7 \epsilon D^{2}$; then taking $\epsilon=$ $\delta^{2} /\left(7 D^{2}\right)$, we get

$$
P\left(d\left(A_{t}, G\right)^{2} \geq \delta^{2}\right) \leq e^{-\delta^{4} T / 98 D^{4}},
$$

So

$$
P\left(d\left(A_{t}, G\right) \geq \delta\right) \leq e^{-\delta^{4} T / 98 D^{4}} .
$$

Thus, the probability of $d\left(A_{T}, G\right)$ being bigger than $\delta$ goes to zero exponentially fast. 


\section{ACKNOWLEDGMENTS}

We thank A Ivaro Sandroni and R ann E I-Yaniv for pointing out mistakes in earlier versions as well as suggesting improvements.

\section{REFERENCES}

A uer, P., Cesa-Bianchi, N ., Freund, Y ., and Schapire, R . (1995), "G ambling in a R igged Casino: The A dversarial M ulti-A rmed Bandit Problem," in 36th Annual IEEE Symposium on Foundations of Computer Science, N ovember.

Banos, A . (1968), “On pseudo-games," Ann. Math. Statist. 39, 1932-1945.

Blackwell, D . (1956a), "Controlled R andom Walks," invited address, Institute of M athematical Statistics M eeting, Seattle, WA.

Blackwell, D. (1956b), "An A nalog of the M inimax Theorem for Vector Payoffs," Pacific J. Math. 6, 1-8.

Cesa-Bianchi, N., Freund, Y ., Helmbold, D., and Haussler, D. (1993), "H ow to U se Expert A dvice," in Proceedings of the 25th ACM Symposium on the Theory of Computing, pp. 382291.

Chung, T. H. (1994), "A pproximate M ethods for Sequential Decision M aking U sing Expert A dvice," in Proceedings of the 7th Annual ACM Conference on Computational Learning Theory, pp. 183-189.

Cover, T. (1991), "U niversal Portfolios," Math. Finance 1-29.

DeSantis, A., M arkowski, G., and Wegman, M. (1992), "L earning Probabilistic Prediction Functions," in Proceedings of the 1988 Workshop of Computational Learning Theory, pp. 312-328.

Easley, D ., and Rustichini, A . (1995), "Choice Without Beliefs," unpublished manuscript.

Feder, M., Mehrav, N., and Gutman, M. (1992), "U niversal Prediction of Individual Sequences," IEEE Trans. Inform. Theory 38, 1258-1270.

Foster, D. (1991), "Prediction in the Worst-Case," Ann. Statist. 19, 1084-1090.

Foster, D ., and Vohra, R . (1993), "A R andomized Rule for Selecting Forecasts," Oper. Res. 41, 704-707.

Foster, D., and Vohra, R. (1998), "A symptotic Calibration," Biometrika 85, N o. 2, 379-390.

Foster, D. and Vohra, R. (1997), "Calibrated Learning and Correlated Equilibrium," Games Econ. Behavior 21, 40-55.

Freund, Y ., and Schapire, R . (1995), "A D ecision-Theoretic Generalization of O n-L ine L earning and an Application to Boosting," in Proceedings of the Second European Conference on Computational Learning Theory.

Freund, Y ., and Schapire, R. (1996), "Game Theory, On-line Prediction and Boosting," unpublished manuscript.

Fudenberg, D., and Levine, D. (1995), "U niversal consistency and cautious fictitious play," J. Econom. Dyn. Control 19, 1065-1089.

Fudenberg, D., and Levine, D. (1999a), "A n Easier Way to Calibrate," Games Econ. Behavior 29, 131-137.

Fudenberg, D., and Levine, D. (1999b), "U niversal Conditional Consistency," Games Econ. Behavior 29, 104-130. 
Hanan, J. (1957), "A pproximation to Bayes R isk in Repeated Plays," in Contributions to the Theory of Games of Games, (M. D resher, A. W Tucker, and P. Wolfe, Eds.), Vol. 3, pp. 97-139. Princeton, NJ : Princeton U niv. Press.

H aussler, D., Kivinen, J., and Warmuth, M. (1995), "Tight Worst-Case Loss Bounds for Predicting with Expert Advice," in Computational Learning Theory: Second European Conference, EUROCOLT '95, pp. 69-83. Berlin/N ew York: Springer-Verlag.

$\mathrm{H}$ art, S., and Mas-Colell, A. (1996), "A Simple Adaptive Procedure Leading to Correlated Equilibrium," unpublished manuscript.

Irani, S., and Karlin, A . (1996), "On-Line Computation," in Approximation Algorithms for NPHard Problems (D orit Hochbaum, E d.), Boston: PW S-K ent.

Kivinen, J ., and Warmuth, M . (1993), "U sing Experts for Predicting Continuous O utcomes," in Computational Learning Theory: EURO COLT '93, pp. 109-120. Berlin/N ew York: SpringerVerlag.

Lehrer, E. (1997), "A pproachability in Infinite Dimensional Spaces and an Application: A U niversal A Igorithm for Generating Extended Normal N umbers," unpublished manuscript.

Littlestone, N., and Warmuth, M. (1994), "The Weighted Majority A Igorithm," Inform. and Comput. 108, 212-261. [A Iso appeared in the 30th FOCS conference, 1989.]

Megiddo, N. (1980), “On Repeated Games with Incomplete Information Played by NonBayesian Players," Internat. J. Game Theory 9, 157-167.

Vovk, V. (1990), "A ggregating Strategies," in Proceedings of the 3rd Annual Conference on Computational Learning Theory, pp. 371-383.

Vielle, N. (1992), “Weak A pproachability," Math. Oper. Res. 17, 781-791. 